Journal of Engineering and Applied Sciences 15 (1): 33-47, 2020

ISSN: $1816-949 \mathrm{X}$

(C) Medwell Journals, 2020

\title{
Exploring Game Engine Through Malaysian Culture Visual Symbiosis
}

\author{
${ }^{1}$ Dahlan Bin Abdul Ghani, ${ }^{1}$ Irfan Hafizi Bin Ab Latiff and ${ }^{2}$ Luqman Zulhilmi Bin Abdul 'Alim \\ ${ }^{1}$ Malaysian Institute of Information Technology, University Kuala Lumpur, \\ 1016 Jalan Sultan Ismail, 50200 Kuala Lumpur, Malaysia \\ ${ }^{2}$ Faculty of Media Creative, Limkokwing University, Inovasi 1-1, Jalan Teknokrat 1/1, \\ Cyberjaya, L63000 Cyberjaya, Selangor, Malaysia
}

\begin{abstract}
Malaysian children and teenagers or what we call the younger generations, lately have been exposed and flooded by the huge and vast market of $2 \mathrm{D}$ and $3 \mathrm{D}$ games. Though within the huge market and selection of all kinds of variety and genre of games, $<1 \%$ of those games are locally developed games. Lately with the rises of local 3D animation industry, it's not far fetch to raise the awareness of developing 3D games scene in Malaysia. The focus of this research is to look into the development of 3D games that includes the usage of Malaysian culture iconic visual styles such as backgrounds, lifestyles, scenery, common everyday objects, character archetypes and narrative (storytelling). Also, to includes local real-world situation as a situation and the mission of the games itself. Therefore, we hope that this research will benefit the younger generation by highlighting the meaning and importance of Malaysian culture.
\end{abstract}

Key words: Real-world, character archetypes, scenery, 3D animation, Iconic visual styles, culture

\section{INTRODUCTION}

What exactly is a $3 \mathrm{D}$ games or video games in general? A 3D game is a computer-controlled game where players interact with objects displayed on a screen for the sake of entertainment. A video game is essentially the same form of entertainment but refers not only to games played on a personal computer but also to games run by a console or arcade machine. The term "video game" also includes games which display only text or which use other methods such as sound or vibration as their primary feedback device, or a controller (console games) or a combination of any of the above. Even though there are a lot of controversy around video games, there are evidence that support video games as a working and efficient way to stimulate the learning ability of a children (Iman, 2011). Video game help develop a children's brain reflex, decision making and problem solving, also, help them to focus on a subject that may be a bore to them in other medium. That's what this project research aimed for, to produce and develop a game that includes Malaysian identities and values that can achieve the level of integrity mentioned which is helping a children's learning process and development. Prof.O is an idea of a game based on Malaysia. The basic idea behinds it is revolved around the main character itself, Prof.O who is a genius local Malaysian boy that can invent various things and gadgets just from everyday objects. His full name is Omar Daud but that's not where the name Prof.O comes from. Prof.O derived from Prof. "Otak" which means brain in English. In this game, the player will have to help Prof.O in his adventure by solving his problem. The issues and mission that will be touched in this game are local issues such as school bullies, kidnapping and others. This game target to provide several paradigms of edutainment that includes local Malaysian identities in its entireties.

The most difficult aspects in making a video game is the engineering aspects of it or more specifically in this case, its computer engineering requirements. In the past, game engineering was mainly about low-level optimization, writing code that would run quickly on the target computer, leveraging clever little tricks whenever possible. But in the past 10-20 years, games have grown exponentially in complexity. Now the primary technical challenge is simply getting the code to work to produce an end result that bears some semblance to the desired functionality. To the extent that we optimize, we are usually concerned with high-level algorithmic choices. There's such a wide variety of algorithms to know about, so much experience required to implement them in a useful way and so much work overall that just needs to be done, that we have a perpetual shortage of qualified people in the industry. Producing a game today is a very different than it was even in the 90's. Certainly, it's more difficult. In order to talk about specifics, I've classified the difficulties into two categories: problems due to overall project size and complexity and problems due to highly domain-specific requirements. Though this will help me introduce the situation in stages, the distinction

Corresponding Author: Dahlan Bin Abdul Ghani, Malaysian Institute of Information Technology, University Kuala Lumpur, 1016 Jalan Sultan Ismail, 50200 Kuala Lumpur, Malaysia 
between the two categories is a bit artificial, we will come full-circle at the end, seeing that there are fundamental domain-specific reasons (problems due to highly domain-specific requirements) why we should expect that games are among the most complicated kinds of software we should expect to see (problems due to overall project size) and why we should not expect this to change for the foreseeable future. That's what Blow (2014) perceive in his book. On consoles (PS4, Xbox, etc.), the console developer as well as one or two third-party companies will provide some development and editing tools (compiler, debugger, profiler, etc.). Console life cycles, however are about 5 years long and there isn't much motivation for the tool-maker to improve their products toward the end of that cycle. Typically, a console developer will be using an environment with only one to four years of maturity, not an enviable situation. To build game content like 3D meshes and animations from scratches, we use programs like Maya or Blender. However, these programs were originally created for people who make non-realtime, so, they present a poor fit. Lately as games have become a bigger business, the makers of these tools have begun to pay more attention to us, to the point that they put "games" at the top of the list of their product's relevance. But these tools are so deeply rooted in the "wrong area," and so big and slow to change that they still represent something very different from what we really need. For example, most game studios would benefit from the ability to build large continuous 3D world meshes with multiple artists working on the same mesh at once or methods of editing triangular meshes to ensure that cracks and holes do not appear. This would be much more interesting to us than much of the functionality these vendors develop and tout such as sophisticated cloth simulation (Bergen and Gregorius, 2010). In the pasts, the situation with regard to asset management tools has also been poor. A modern game studio needs a fast and robust system for networked revision control of source code, 3D models, animations, sound effects and all the other various data files involved in a game. Lately, some companies have risen to provide asset control specifically for game projects. These tools are still far from ideal but we have reason to hope that they will improve. We also have a lot of workflow problems that are not so, directly tied to specific tool software. On the programming side, our compile/edit/debug cycles are usually far too long. Many games take half an hour or longer to compile when starting from scratch or when a major $\mathrm{C}++$ header file is changed. Even smaller changes, causing a minimal amount of recompilation and relinking, can take as long as two minutes. In general, $\mathrm{C}++$ seems to encourage long build times.
Once the build time has grown wider and wider, a team may end up putting a significant amount of work into refactoring their source code to make it build more quickly. Often this happens too late as the spaghetti of file dependencies has become, so, severe that fully refactoring it would be akin to restructuring the project from scratch. In fact, the best way to avoid long build times is to architect the entire code base to minimize dependencies. This does not happen too often because many studios do not take these workflow issues as seriously as they ought to as the effect of the problem is somewhat intangible and there are always, so, many clear and present issues to deal with or they don't have sufficient discipline to deal with such a subtle issue over periods of time measured in years. Once the game is compiled, a test must be run check any changes in variable used in the code. However, startup times can be very long, since, games often need to load large amounts of data. Startup time can typically be three minutes for a debug build with large data files for which load-time optimization has not been done. Add this to the compile-and-link time and you can easily have a 5 min delay between making the smallest possible code change and seeing the new version of the game running. Testing the actual change will take longer as the programmer needs to set up the proper conditions within the game world to exercise that code path. Visual $\mathrm{C}++$ provides an "edit and continue" feature wherein one may splice code changes into a running program and avoid these delays. However, this feature doesn't work reliably enough to eliminate the problem (though when it does work, it is very welcome). This feature is not usually present in the compiler environments for console systems. Another way to avoid this turnaround time is to write a significant amount of your code in a higher-level extension language that can be dynamically reloaded by the game engine without restarting. There's an analog issue for the content development parts of the team with regard to how long it takes them to see the effect of changing a texture or model. Fortunately, this problem is easier to solve as loading these assets is handled entirely by our game engines, we are empowered to fix the situation. Currently, some game engines written by experienced developers provide automatic reload of content resources at runtime which is becoming a more widespread trend. Once, we have done all this, we need to worry about stiffness, the fact that merely by adjusting constants, you can cause the simulation to become unstable. To the best of our current methods, good integration techniques can only provide an area of stability within the simulation space, you must take care not to step outside that area. This becomes much more complex when we consider all the platform available for games. Just imagine all the different 
consoles, PC games and mobile games currently in market how difficult it is to standardized the process for developing games in all across these platforms. Though with current modern technologies, there are several tools that allows developer to develop games with graphic or 3D animation knowledge only. In Unity game engine, there custom assets that allows developer to create all the interactives element of a game that includes dialog, cutscene, object interactivity with drag and drop functions. While it does require the developer to have basic knowledge on how game system works, it sure is easier than having to code a game from scratch. Developing a game minus the coding parts could save countless of hours in the development part. While sure, developing a game with this kind of assets may have its limitation but using it properly, can bring out the full potential of this assets. This Unity engine asset is called, Adventure Creator.

Literature review: First of all, lets explain a bit about Malaysia. Malaysia is a country that mixes tradition and modern society seemingly with ease. Although, many traditions have been maintained from their historic culture, many people have a way of life that more closely reflects that of modern urban society found elsewhere in the world. The country of Malaysia is very diverse and a significant number of people live in more rural areas, though, nearly three quarters of Malaysia's population live in urban settings (DSM., 2010). No matter the location, Malaysia seems to be a rapidly progressing and changing country as technology is driving the people forward. In this setting competition is rising and parents strongly encourage their children to succeed but not with the societal pressure found in some Far Eastern countries. It is the children that seem to come first for many in Malaysia as this is truly the center of most people's worlds and remains at the heart of the culture. Speaking of culture, multi-racial country like Malaysia with Malay, Chinese and Indian is an example of a modern cultural assimilation civilization that have nurtured throughout the years to birth a new kind of unique lifestyle (Alcazaren, 2011). That's the kind of value that we aim to symbiote with the game that we are developing in this project. For a starter, Malaysian identities can be visualized in a video game by the environment and the vibe of the game itself. The setting and background of the game that's designed based on Malaysian demographic and geographic can give the audience a sense of home and immersion as to where the game take places. Not only that, everyday objects including clothing styles and archetypes can also be implemented as to further convinced the audience with the same local Malaysian vibe and values. Furthermore, by adding the content of the game or the mission of the game based on what actually happens in reality of
Malaysian life will definitely seal the deal in giving the audience the senses of Malaysian in the game while playing. Senses of relation in video games to real life can provide a mental support to its audience as stated in an article titled "How Video Game Can Teach Your Brain to Fight Depression" (McGonigal, 2015) thus, providing an effective way of showcasing Malaysian identities and values in this project.

First and foremost, maybe people will ask why develop a game to promote cultural values and identities when the same can be done with mobile application or more widely known as "Apps". That may be true but game and apps have a very different impact to its audience based on the content itself and the approach that it conveys to its audiences. First of all with video games, it can target broader audiences. Rather than targeting children only, we can target the younger generation as a whole target, since, these wide range of younger generation age gap are more prone be interested in video games. This also impact the brain development efficiently than an Apps., since, its more interactive with its audience, therefore, creating more stimulus catalyst for the brain as they are playing (HPPST., 2012). Children and the younger generation of today have an increasing attraction towards electronic media in their play. With video games, phones and the internet in abundance, if such leisure activity is impacting their cognition or academic performance or whether it would be more beneficial to read through pages of information in an apps, surely video game is the more leisure choice of that. After a busy day, children do need downtime to rest and relax. Increasingly kid's leisure time is spent gaming. Historical research shows in some cases that interactive gaming can have positive effects for cognition by promoting memory, attention and reasoning. Other speed oriented games have been shown to improve perception and motor skills, so should gaming for relaxation be encouraged? This type of leisure activity produces a 'transfer effect' influencing learning processes thus improving student performance at school. With an emphasis on gaming and reading they linked patterns of leisure activity with performance in phonology, reading and comprehension, math, long term memory and reasoning. Fascinatingly gaming previously thought to improve fluid intelligence showed little or no positive correlations to performance whilst reading did, particularly in memory and comprehension. It seems then despite lack of a causal link that reading may be more likely to enhance academic performance. Should we assume that time spent gaming and away from homework is harmful to students? A further comparison of reading through information in an applications and gaming to most frequent leisure activities showed no negative 


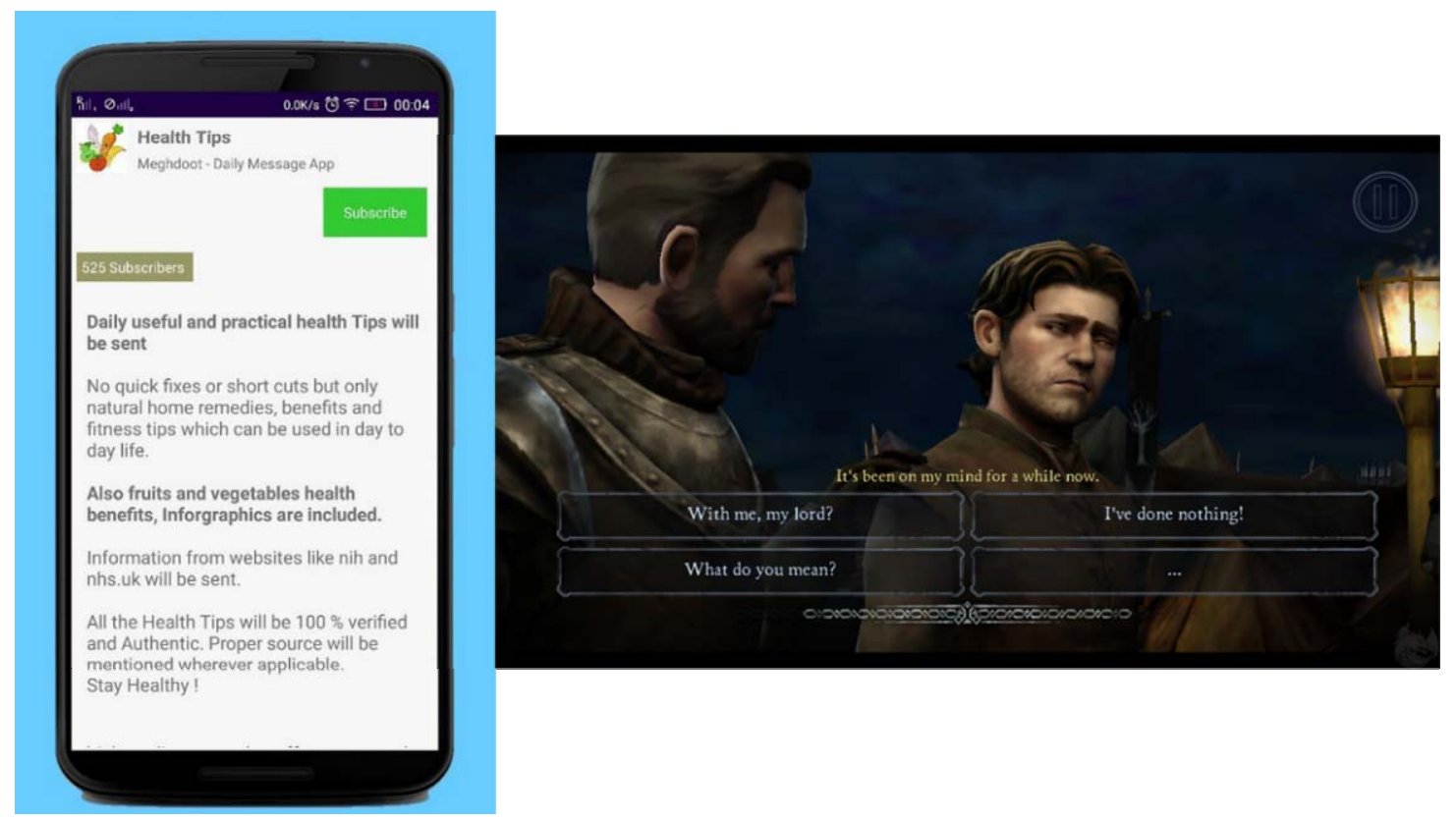

Fig. 1: Difference of getting information through apps and interacting in game with dialog sequence and multiple choices

patterns but interestingly resting had a favorable effect on performance as well as reading. So, frequent leisure activity is not necessarily harmful to progress or always at the expense of homework but can be enriching. We think that video games are mainly recreational activities and the cognitive stimulation provided is very different from school learning. But ultimately, if it's used right with the right content, the right approach, video games is ultimately better choice for this research project than application (Fig. 1).

As for the literal development part itself, both game development and application development are similar with one another with some obvious differences. To set it straight, the similarity of developing both these things is that it requires sophisticated knowledge of programming language to code an effective and responsive application or video game. It's also similar in terms of its approach in the GUI or Graphical User Interface. Proper research needs to be done for both games and apps in order to bring the user the best experience in interactive and innovative user interface experience. And it could mean whole lot different between a half-done user interface with a well-researched interface. This is because with the graphical user interface, user somewhat communicate directly with the medium in use, in this case, in games or in applications. Perfect graphical user interface would be the one that appears to be assisting its user without being a hindrance and an eyesore. Wrong sizes or maybe color of a graphical user interface can cause them from being the assistance to the program to becoming a hindrance and useless to the user (Fig. 2).
Video game development is the process of creating a video game. Development is usually undertaken by a game developer which may range from a single developer to a global team of developers. Traditional commercial PC and console games are normally funded by a publisher and can take several years to develop. Indie games can take less time and can be produced at a lower cost by individuals and smaller developers. The independent game industry has seen a substantial rise in recent years with the growth of new online distribution systems (Serebrin, 2017) such as Steam and Uplay as well as the mobile game market, such as for Android and iOS devices. For this project in particular, Unity3D game engine was chosen as the medium to "realized" the game in progress. The biggest factor in this choice is the beginner friendly approach that Unity game engine propose to their audience. Not only that, they have a free option to their software with almost full features only lacking several professional tools which are not that big of a difference for a beginner. Also, the vast collection of assets in the assets store allow user to pick which one is the most suitable to use for their respective project in mind. A wide collection of tutorial archive on their websites also helps a lot for anyone wishing to start developing a game. Furthermore, for developer who don't have any experience in coding a program or any knowledge in programming language at all, they can still start with Unity. This is because there are assets in their assets store that allows developer to "code" the interaction and interactivity of the game through drag and drops functions. Not only this makes it easier for the developer 


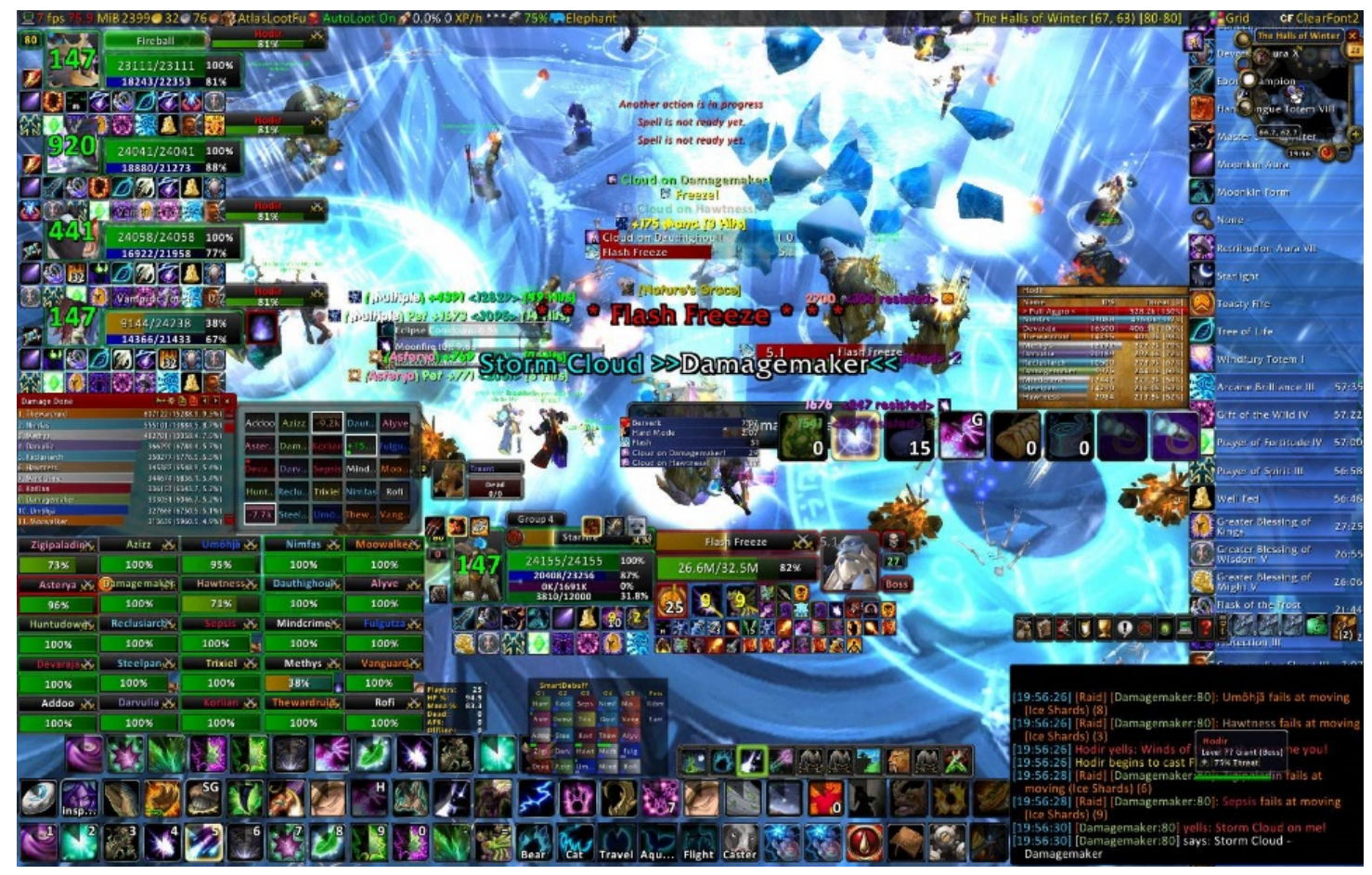

Fig. 2: Example of a bad graphical user interface in video games

to produce a game but this can also save countless of hours in coding and debugging the game. This particular asset is called Adventure creator that is available in the Unity assets store for the cost of $70 \$$. Some of the features of this asset provide support for variety of cross-platform including Android and IOS, support for 2D, 3D and even 2.5D games and the biggest feature, visual scripting workflow. That's the feature mentioned above where user can code the game only by drag and drop functions.

Culture is part of human development which evolves with civilization from ancient times to today's digital era. Video games can contribute to the cultural industry through signs and symbols and is captured from people's different lifestyle and various grounds including religion, belief systems, politics, tradition, language, tools, costumes, buildings and arts. Now, of course integrating cultural identities into video game will not be an easy task as extensive research needs to be done to identify proper way to merged the two together. But if done properly, conveying message of culture, lifestyles, socio-identity of Malaysian can be done effortlessly and smoothly as it transitions through the gameplay itself. One of the most important things to study for integrating video games with Malaysian identities is the environment and background settings in the game. Matching building architectures, everyday surrounding objects such as vehicles, or even bus stop designs, can affect the overall vibe of the game. One more thing to consider is the clothing or costume of the main character and other characters in the game must follows the correct socio-identity of Malaysian (Fig. 3).

When you pick up and begin to read a book or watch a movie, the characters in the story are inherently formless. The first time you run into a character, you only have a few distinct descriptors to hold onto- "a tall redhead," "a stern-looking man." As you continue to read and the character takes more action in the story, more little pieces of description are pushed your way and you can gradually shape a picture of a human being based on the context that is given to you. And that's why character design is important. Character is what drives the story and breathe life into a film (Pixar, 2012). For this research project, Prof.O, we've decided the protagonist or the main character of this game will be a 10 years old genius Malaysian boy have the ability to tinker parts and objects into usable weapon or gadgets to assist him in his adventures. Right away, we decide to give him a distinct feature of an afro hair to make him standout. In this early stage, we only just decided on his key physical appearance. For the next step in the designs, socio-identity of Malaysian will be integrated in his designs including his gadgets, clothing and as far as suitable catchy catchphrase (Fig. 4). 


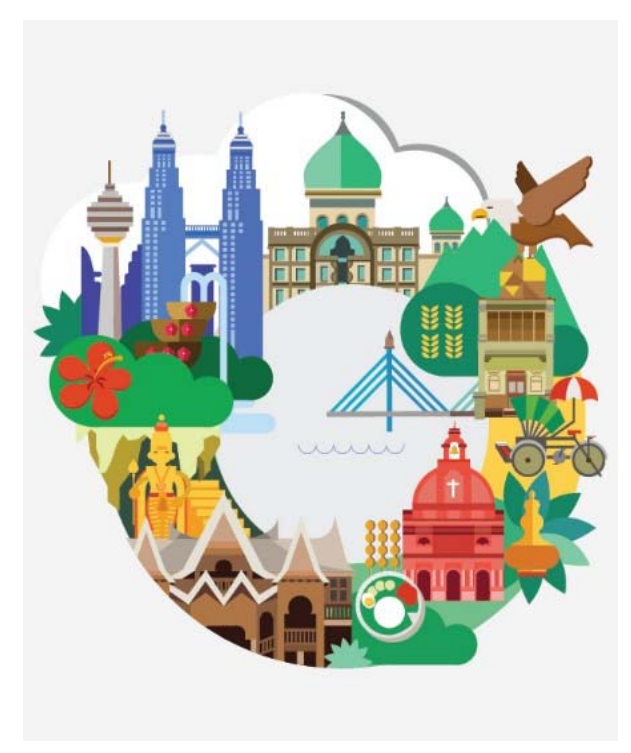

Fig. 3: Example of iconic symbols of Malaysian identities
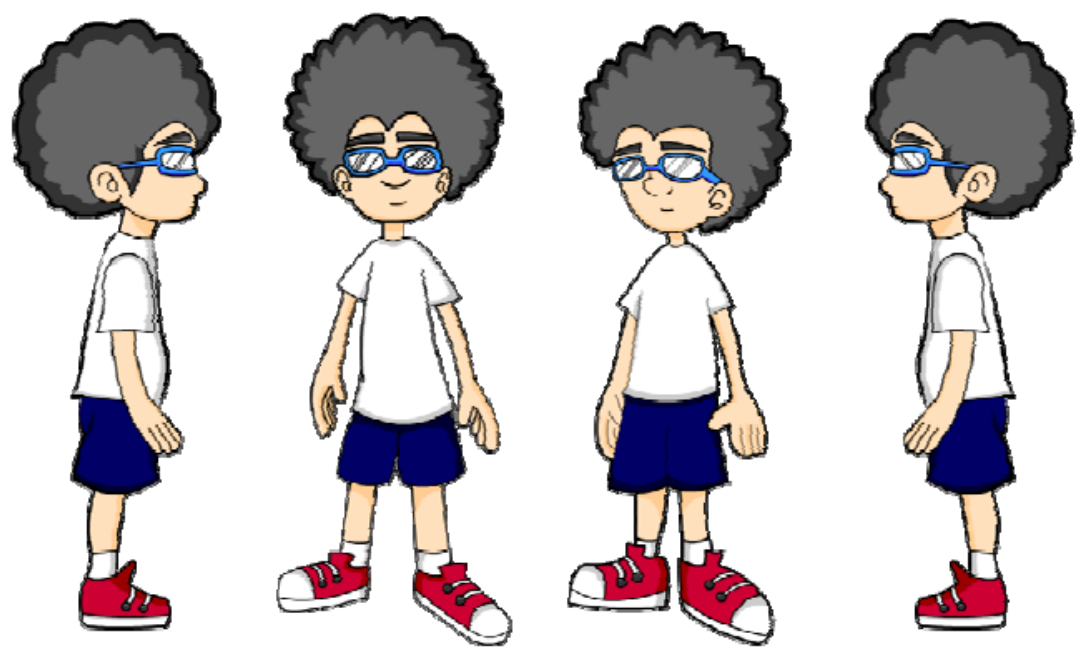

Fig. 4: Early drafts of Prof.O character designs

Overall, the objective of this study was to develop a $3 \mathrm{D}$ game integrated with Malaysian identities and values. To do this, the first step was to explore the subject through relevant literature and analyzing proper designs and culture about Malaysia. A lot was learned from the investigation that was conducted during the first part of the study and I am glad that I decided to focus much of the time on this part instead of rushing to the actual production. Reading literature by artists and developers in the industry who explained their procedures and processes of developing video game and designing character based on the main content needs, equipped me with new ideas and views on how to approach the task prior to the actual production.

\section{MATERIALS AND METHODS}

Research methodology is a guidance or technique that is used by researcher in order to carry out the project research and studies for the project to proceeds smoothly from the start, to the end (Kumar, 2011). The purpose of research is to inform action (UFS., 2009). Thus, your study should seek to contextualize its findings within the larger body of research. Research must always be of high quality in order to produce knowledge that is applicable outside of the research setting. Furthermore, the results of your study may have implications for policy and future project implementation. Ultimately, the key to a successful research project lies in iteration: The process 

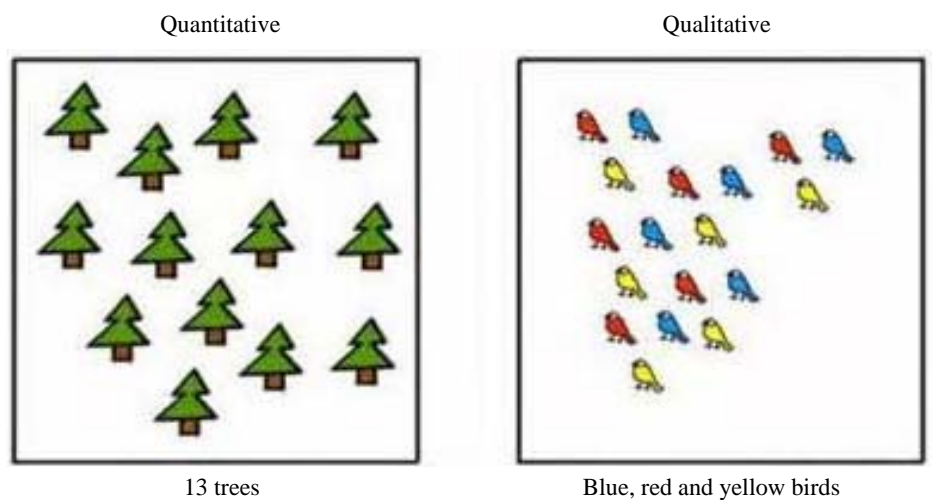

Blue, red and yellow birds

Fig. 5: Simple visual representation of quantitative and qualitative

of returning again and again to the research questions, methods and data which leads to new ideas, revisions and improvements. It is easy to think of research as a step-by-step " $1,2,3$ " process but it is important to be flexible and open to change. Oftentimes by discussing the research project with advisers and peers, one will find that new research questions need to be added, variables need to be omitted and other changes need to be made. As a proposed study is examined and reexamined from different perspectives, it may begin to transform and take a different shape. This is to be expected and is a component of a good research study. Basically, the purpose for this research is to assists in the development of a 3D game, "Prof.O". The basic idea behinds it is revolved around the main character itself, Prof.O who is a genius local Malaysian boy that can invent various things and gadgets just from everyday objects. His full name is Omar Daud but that's not where the name Prof.O comes from. Prof.O derived from Prof. "Otak" which means brain in English. In this game, the player will have to help Prof.O in his adventure by solving his problem. The issues and mission that will be touched in this game are local issues such as school bullies, kidnapping and others. As mentioned the above, video games development is still lacking the support and the encouragement that it deserves in Malaysia. That's the main purpose of this research, to encourage the development of 3D games development in Malaysia as well as developing a 3D games in this project called Prof.O. For this research, data are accumulated via game testing which can be used to measure game quality (Ramadan and Hendradjaya, 2014) and a simple questionnaire for the feedback of the player at the end of their gameplay which will be conducted to a sample of target audiences. Particularly, this project target audience will be on the younger sides ranging from young children of 10 years old age until 16-17 of ages. Though, the content that will be portrayed in this game will be suitable for all age ranges.
There are numerous sources that will be used to find information about the subject or project of matters, Prof.O, 3D games development. The following will elaborate more on each of the techniques and sources used for the research. According to University of North Dakota Academical Research Centre, multiple sources of research will further approve the research in hand (UND., 2008). There's always a debate in research method between qualitative research and quantitative research methods. Collecting data to inform and improve website usability is consistently a challenge, for companies from startups to large enterprises. There's a wide range of information and healthy debate on what user experience research methods should be used and when. When it comes to quantitative versus qualitative research, best practices suggest a mix of qualitative and quantitative approaches to effectively capture a more holistic perspective (Wisdom and Creswell, 2013). A challenge, however, is how to combine qualitative and quantitative testing in a cost-effective, productive way to rapidly obtain data to drive user-centered design. For this research in particular, we decided to use qualitative research to start with the precedence of the project. Qualitative testing gives a better picture on the research matter (Anonymous, 2017) as it allows collection of data directly from the target audience in an environment where the target audience experience themselves in the project matter in this case, Prof.O, 3D games project (Fig. 5).

The researcher will issue a game testing session for the target audience in order to receive information and data on several major subject matters, knowledge and understanding of 3D games, their knowledge of 3D games development scenes in Malaysia and the quality of the game developed in this project by the end of this research matter. Game testing, a subset of game development (Kraft, 2010) is a software testing process for quality control of video games. The primary function of game testing is the discovery and documentation of software 
defects (aka bugs). Interactive entertainment software testing is a highly technical field requiring computing expertise, analytic competence, critical evaluation skills and endurance. In recent years the field of game testing has come under fire for being excessively strenuous and unrewarding, both financially and emotionally. Game testing is used mainly for quality assurance (Ruuska, 2015). By the end of this process, a simple interview/questionnaire session will be held with the audience to get their feedback to be processed as our research data/information. By the end of this research also, we target to attain a deeper understanding on the market's trends (Nantes et al., 2008) and requirements prior to the development of the project (Wang and $\mathrm{Wu}, 2015$ ) from having the game testing sessions and questionnaire answered, this is a form of qualitative research.

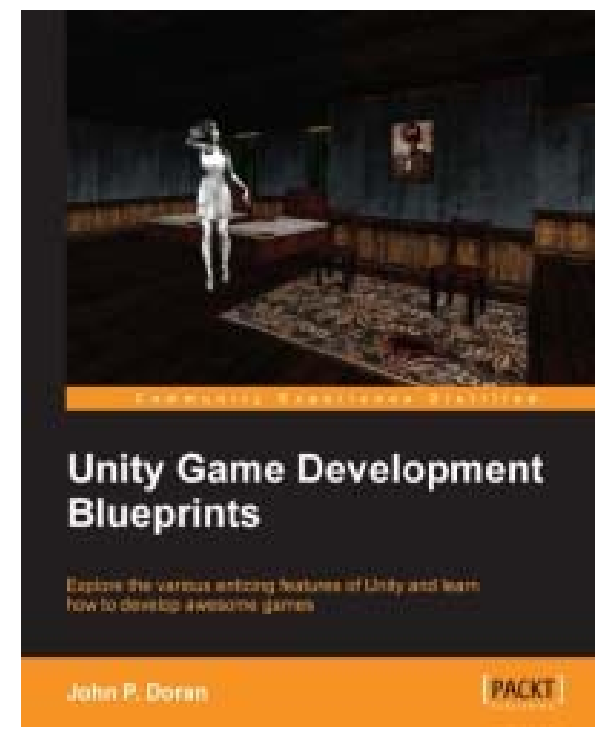

Fig. 6: Example of available e-Books
The internet has unquestionably changed the way we acquire information. In today's digital age, there are sources such as e-Books and e-Journals which are basically real books and journals which have been converted into a digital imperishable form (Abbott, 2014). An e-book is a book in electronic format. It is downloaded to a computer, PC, Mac, laptop, tablet, smartphone or any other kind of reading device and is read on the screen. It can have numbered pages, table of contents, pictures and graphics, exactly like a printed book. e-Books offer many benefits and advantages and this article shows a few of them. It is very simple and easy to purchase and download e-books through the internet. It is exactly like purchasing any other product. The only difference is that after payment you will either be directed to a download page or receive the download link in an email. All you have to do is click on the link and the e-book will automatically download to your computer, to a folder of your own choice. Although, it can be debatable that ebook do have some obvious disadvantages in terms of citation credibility (Kimmons, 2016), piracy and such things, in this particular project research, e-Books and other e-Journals actually makes a difference in terms of research material and assistance in the project development. Furthermore, the Internet has made it possible for users to view a book's or journal's contents using the computer (Byars, 2015). As for this research, we will conduct research regarding this project which is Prof.O 3D game development via. books, journals, websites, reports and articles in order to develop the project (Fig. 6).

As mentioned earlier, the internet itself is essentially a hasty medium to acquire information, particularly for research on videos and other related materials to the topic at hand. For this project, most of the game development process in terms of graphic and technicality are on unity as the medium. For in game contents and processes, adventure creator are used (Burton, 2015) which is in video form uploaded on youtube streaming platform (Fig. 7).

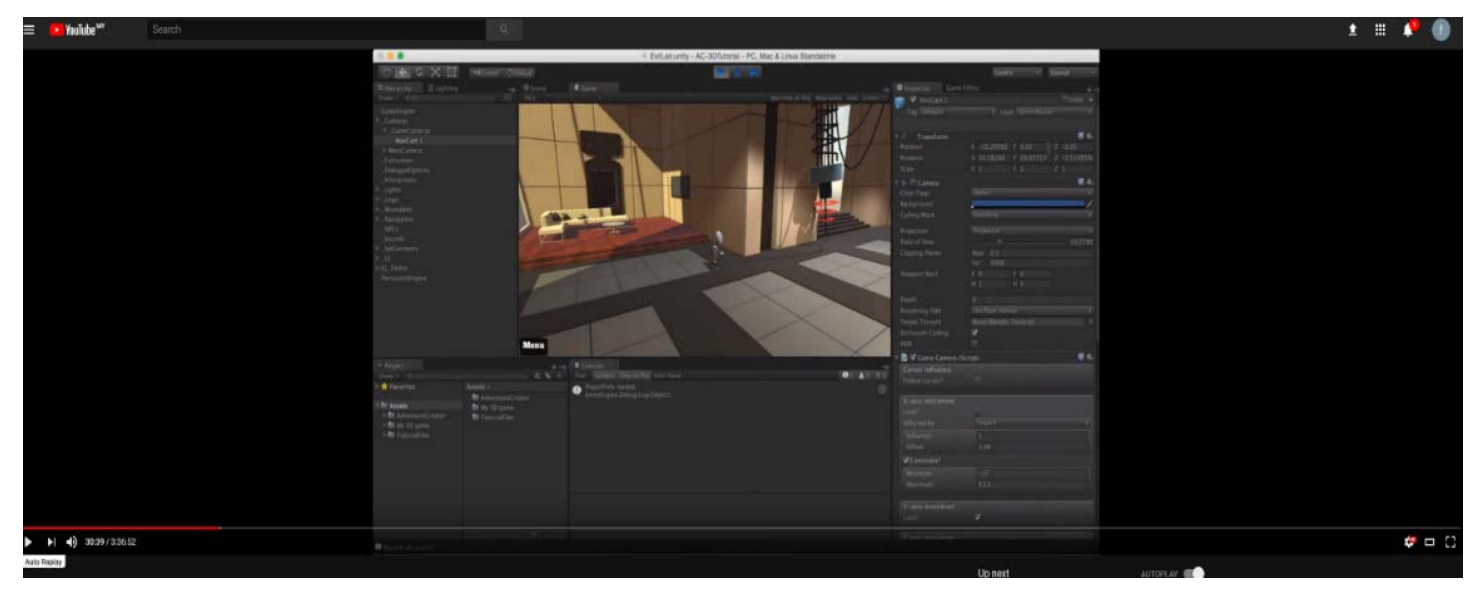

Fig. 7: Tutorial referred above 

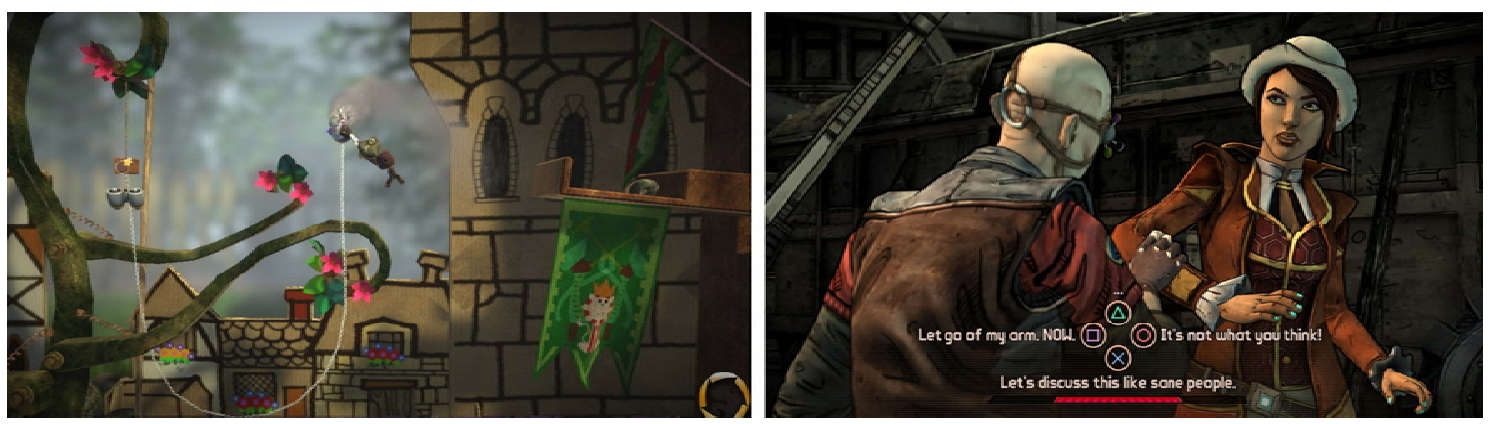

Fig. 8: Little Big Planet and Telltale game, respectively

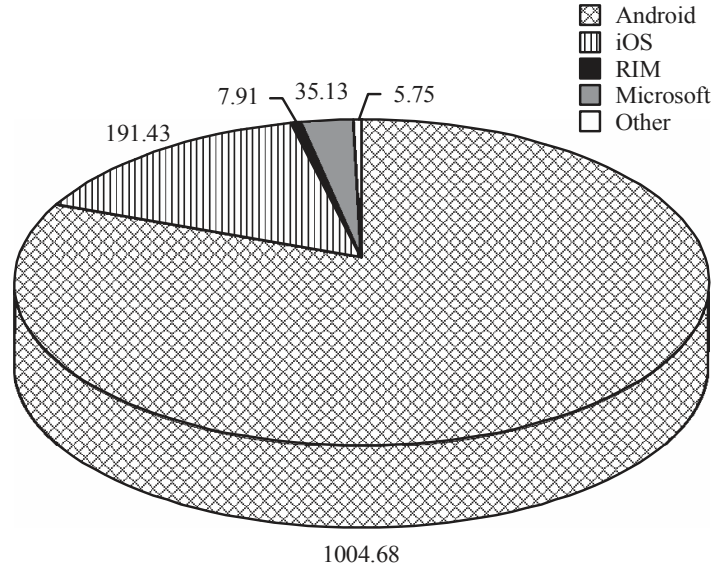

Fig. 9: Statistic of Android compared to other platforms

Not to mention, the internet as a medium helps a lot as referred on Fig. 6, most of research material from e-books, journals, reports and articles were acquired through internet lurking. The impact of internet as an informational platform cannot be underestimated, according to University of Colorado Reports, the World Wide Web is an extraordinary resource for gaining access to information of all kinds including historical and each day a greater number of sources become available online (UCB., 2015). The advantages that the internet offers students are tremendous so much, so that, some may be tempted to bypass the library entirely and conduct all of their research on the web. We as students need to pursue knowledge with every tool available including the internet, so, long as they do so judiciously. It is important to know that the web is an unregulated resource. Many sources on the web have proven to be unreliable, biased and inaccurate checking the reliability and accuracy of information taken from random sites could take more time than going to the library. And using information you have not checked from such sources could have a detrimental impact on your final grade. The key is to learn how to use the web to your best advantage.
There is copious amount of existing games of genre adventure/strategy, some has its own back story and character and some are just plain mind-boggling fun. For console gamer, they must have played or even heard of the game Little Big Planet (Fig. 8). It's a 3D side-scrolling platformer where the player needs to use their surroundings to overcome given obstacle. One might say that this kind of game is categorized as Time-Killer as it's, so, easy to get engrossed in the game environment also not to mention the strategy and gameplay itself. This kind of game helps children or younger audience in developing their reflex and problem-solving skills which can actually be applied into real life as some of the puzzle requires high level of logic and basic common knowledge to solve properly. As mentioned by several authors in their article, playing video games of this genre helps in nurturing cognitive skills (Granic et al., 2014). There also, this same genre of game that takes gaming to another approach. Several years ago, this approach doesn't exist, that is a Telltale game. In this type of game, they use the environment and the storytelling of a game to tell a whole new adventure. It's like watching a movie but the audiences are involved in the decision making, the outcome, the result and the final ending on how the story goes (Taylor et al., 2012). Some good example of this genre of game is the Walking Dead: Telltale series. Those game references act as a guide for the researcher to develop and design the project.

In Fig. 9, for the time being, this project just entails to $\mathrm{PC}$ and android device to run. Though, other platform can be considered in the future. PC and Android were chosen as it's the most common device that any households own at least one of those devices nowadays (Vincent, 2017). Also reported by Google, in 2017 android user is more than 2 billion worldwide (Matney, 2017). For this research, data will be sampled among 10 young adults, also 10 children and teenagers. This is because as mentioned above, these are the main 


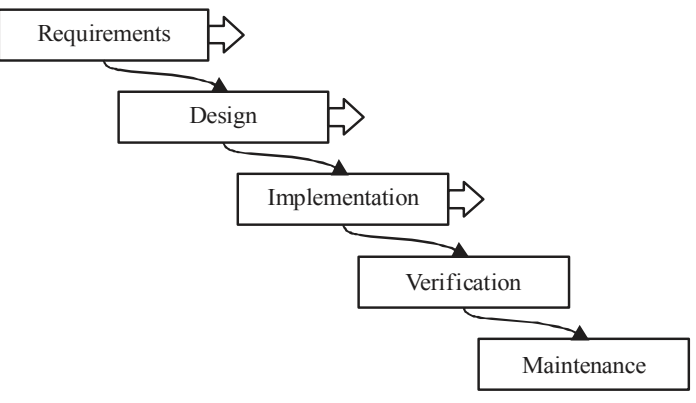

Fig. 10: Standard form of Waterfall Model processes

target audience for this game as it is the most suitable and the most beneficial audience (ABC NEWS, 2011) for this genre of game that this research develops. For the young adult, the age will be ranging around 18-21 years old, as for the children and teenagers, the age will be around 10-17 years old. The reason for choosing this demographic is further supported in a Huffington news report titled, importance of doing puzzles with your kids (Sungar, 2014).

Figure 10 shows the initial step prior to developing the product is the research procedure and technique step. Today, there are countless types of research methods available. As for this study, we have decided to use Waterfall Model (Munassar and Govardhan, 2010) as the methodology and as a guideline. All of the phases in the Waterfall Model will be elaborated accordingly within the project scope. However, there are some modification in this Waterfall Model to best suit the project and research needs. The usage of this model generally depends on the current project's needs and fits. That's why a lot of game developer often use Waterfall Model or Agile Model in game development as it is easily adaptable to many kinds of forms depending on the project type, scope, genre and last but not least its audience. Different kind of audience may be affected if different kind of approach is used. From this technique, the researcher may deduct that every phase will need its own evaluation before proceeding to the subsequent phase in the cycle. The project supervisor will also play a key role in approving each phase's outcome before advancing to the following level of development. To explain it simply, Waterfall Model is the sequential nature of the phases. One phase completely finishes before another phase begins. With a nod from the Supervisor, you continue to proceeds further into the Waterfall Model processes. The Waterfall Model is an ideal choice for smaller, simple projects because it is made of the defined stages that are done sequentially and each stage is fully completed before moving to the next stage (Anonymous, 2016). It is also easy to manage as the process cannot overlap with each other, once a step is completed, just proceeds to the next one. If there's a problem along the way, move up the process back a notch and vice versa (Amlani, 2012).

In the figure above, you can see the basic form of Waterfall model that is widely used across all kinds of development process. The processes move downward in a straight line as it moves towards the next proceeds of a particular project. Basically, it functions as below (Tutorialspoint, 2010).

Requirement gathering and analysis: All possible requirements of the system to be developed are captured in this phase and documented in a requirement specification DOC.

System design: The requirement specifications from first phase are studied in this phase and system design is prepared. System design helps in specifying hardware and system requirements and also helps in defining overall system architecture.

Implementation: With inputs from system design, the system is first developed in small programs called units which are integrated in the next phase. Each unit is developed and tested for its functionality which is referred to as unit testing.

Verification: All the units developed in the implementation phase are integrated into a system after testing of each unit. Post integration the entire system is tested for any faults and failures. Once the functional and nonfunctional testing is done, the product is deployed in the customer environment or released into the market.

Maintenance: There are some issues which come up in the client environment. To fix those issues patches are released. Also, to enhance the product some better versions are.

Though, this particular project; Prof.O, 3D game development will use a modified version of the Waterfall Model based on Manninen's Generic Model (Manninen et al., 2006). The modified version contains an extra step from the usual method. This assure the planning of the project to be more detailed as in its content and its values as this project as mention in chapter 2 above are required to portray cultural and national values in its surround, character archetypes, models, objects, background and its gameplay and overall vibes. In this modified version its listed in Fig. 11.

Concept phase: In which the conceptual design of the game is drafted. Concept art is a form of illustration used 


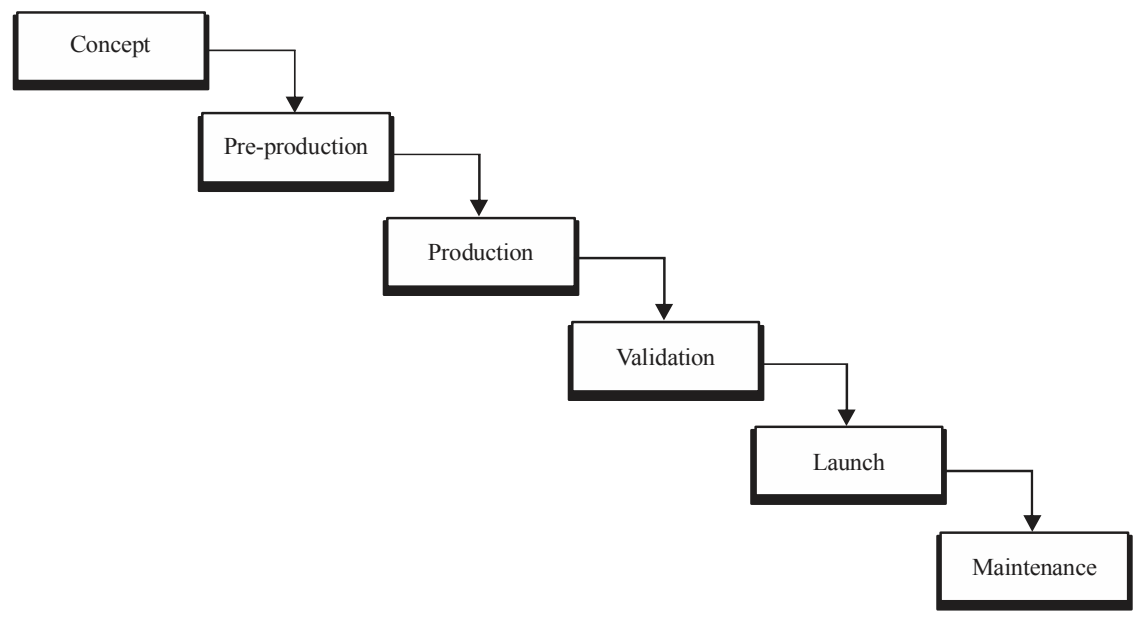

Fig. 11: Modified implementation of waterfall model
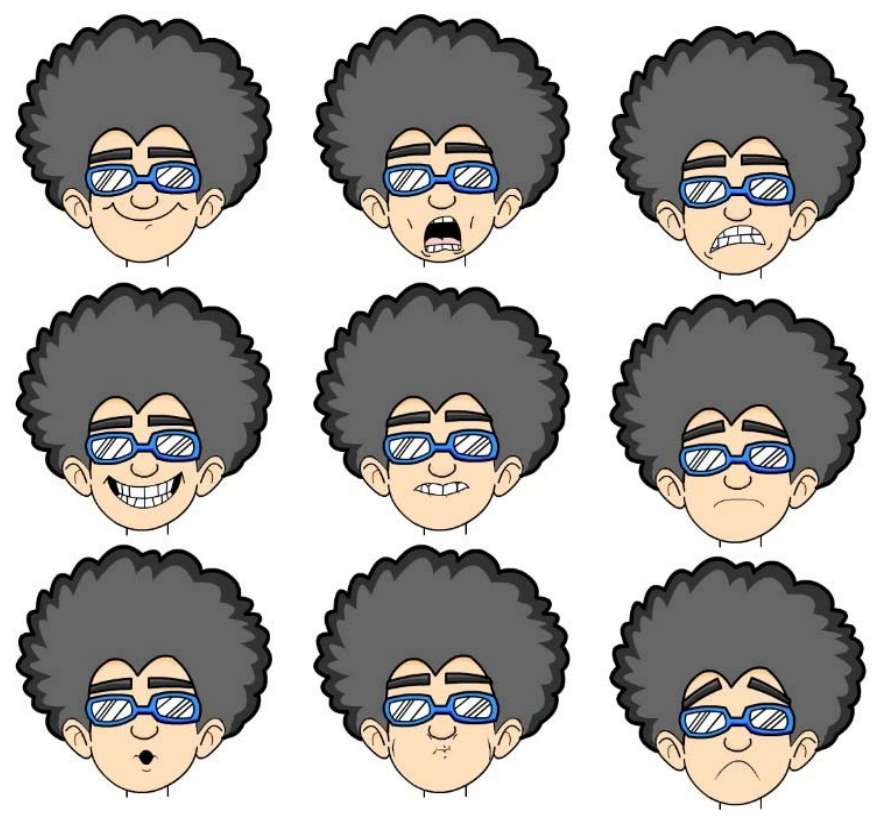

Fig. 12: Concepts arts of Pro.O's facial expressions

to convey an idea for use in films, video games (Janlert and Stolterman, 1997), animation, comic books, or other media before it is put into the final product. Concept art is also referred to as visual development and/or concept design. This term can be applied to retail, set, fashion, architectural and industrial design. Concept art is developed through several iterations. Multiple solutions are explored before settling on the final design (Gray and Julian, 2004). Concept art is not only used to develop the work but also to show the project's progress to directors, clients and investors. Once the development of the work is complete, concept art may be reworked and used for advertising materials (Fig. 12).

Pre-production: Pre-production phase consists of creation of a working prototype. The objective of the phase is to "plan, test and evaluate everything possible". Pre-production generally consumes an amount of time which is proportional to the amount of time you plan to spend actually developing a game but in general, for a 1 year project, pre-production would take between 6-8 weeks (Scacchi, 2012). Pre-production happens in 


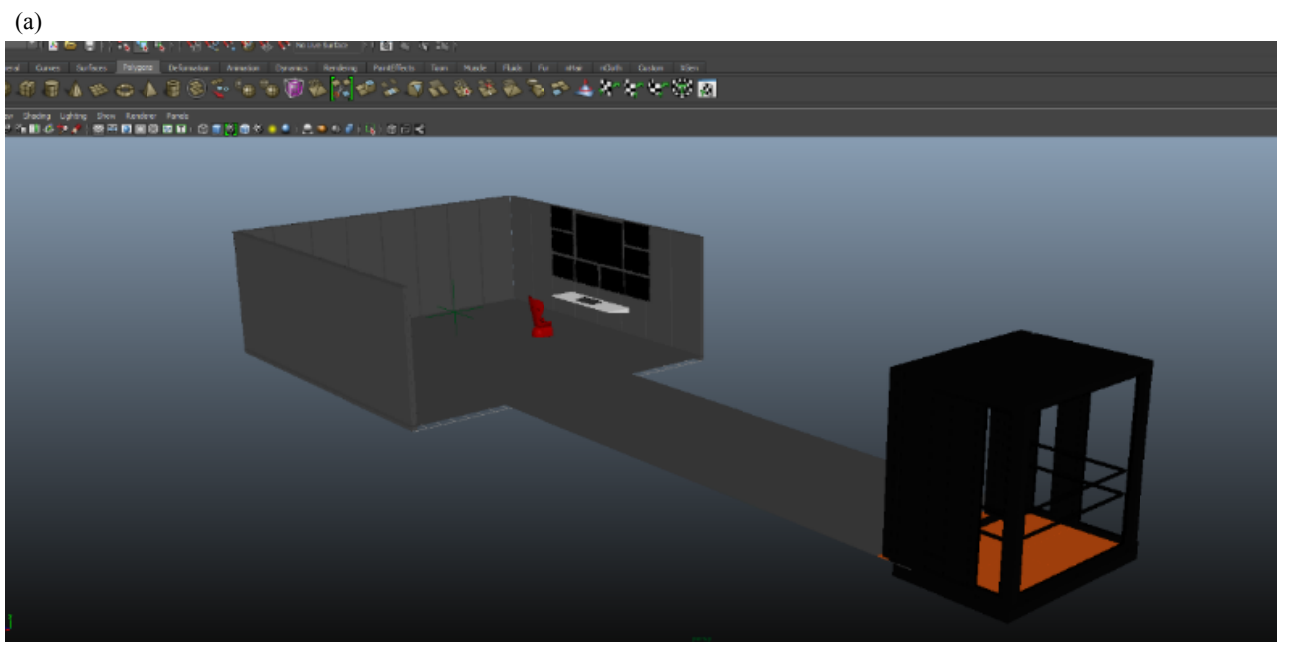

(b)

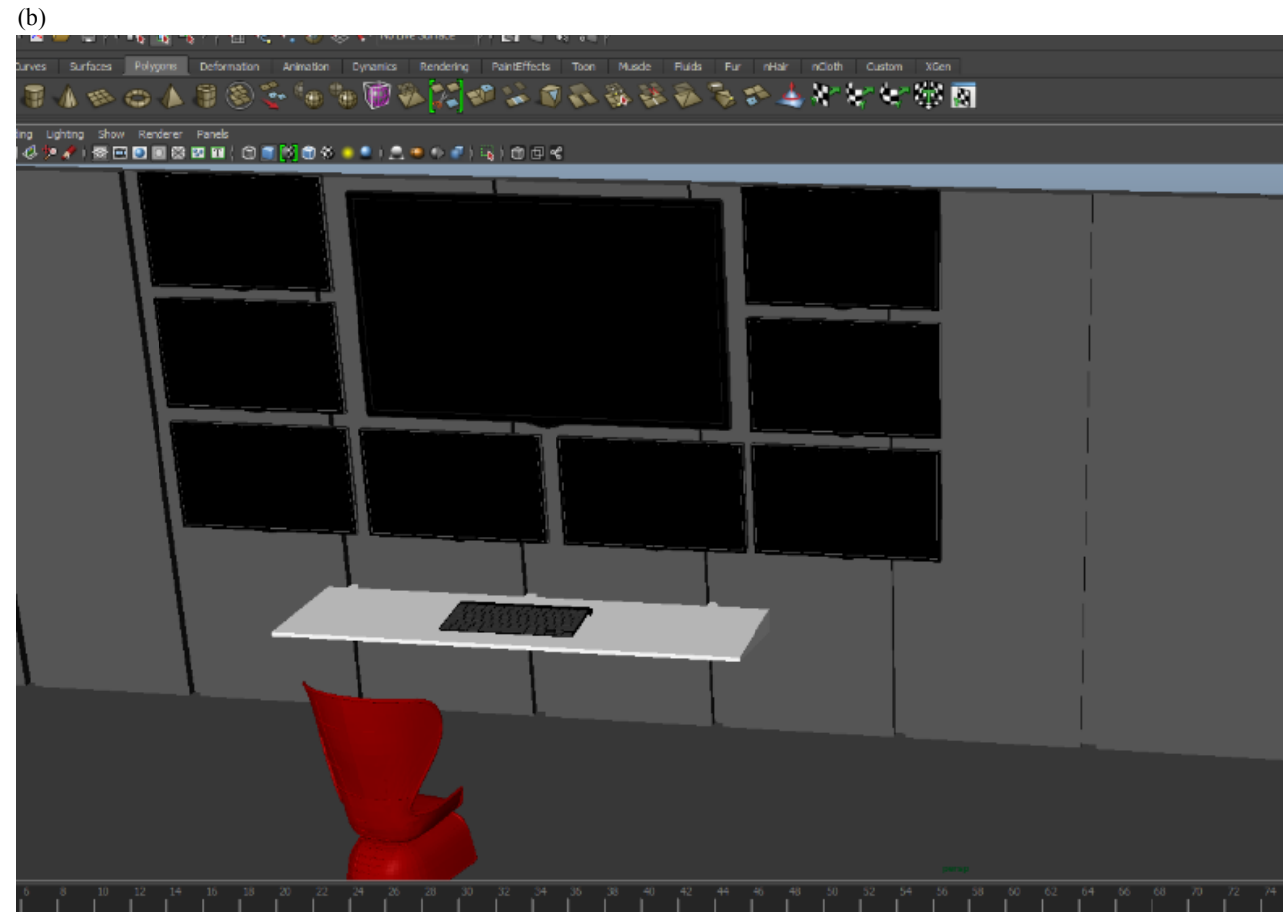

Fig. 13: (a, b) Pre-production part of Prof.O development, modelling and designing environment

steps and needs to be looked at as laying down a framework for what your game is going to be and creating very rigid guidelines for your whole team (Design/Programming/Art/Etc) to follow as they transition out of pre-production and into actual production of the game (Jarvi et al., 2013) (Fig. 13).

\section{RESULTS AND DISCUSSION}

Production phase contains all tasks, from programming to graphics and sounds and integration needed in game creation. Typically, games are produced through the software development process. Games are developed as a creative outlet and to generate profit (Wi, 2008). Development is normally funded by a publisher. Well-made games bring profit more readily. However, it is important to estimate a game's financial requirements such as development costs of individual features. Failing to provide clear implications of game's expectations may result in exceeding allocated budget. For this particular project, budget is planes to be supported by the faculty in charge as it's a project in venture with the faculty itself. Validation and testing phase includes functional testing as well as quality 


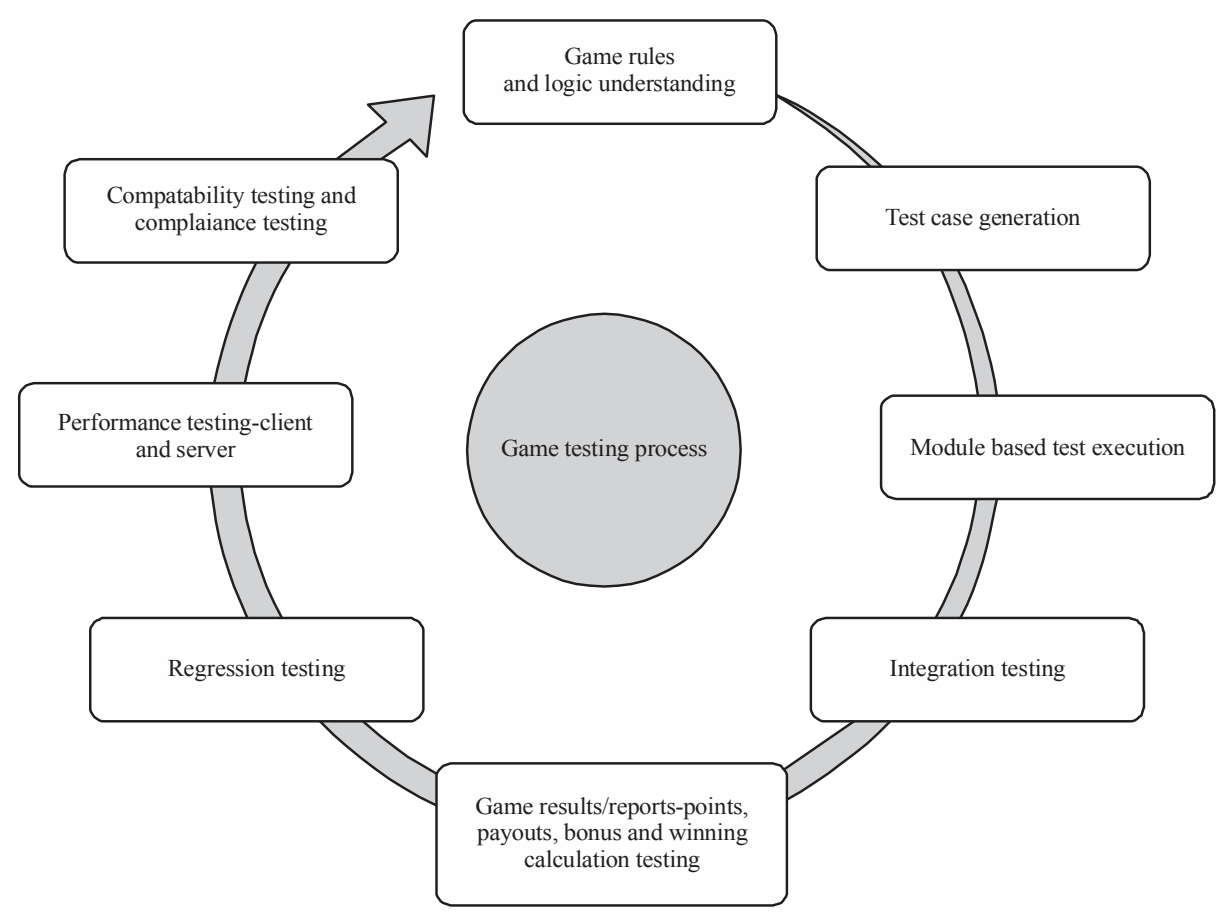

Fig. 14: Process and cycle of game testing methods

assurance of gameplay, user interfaces etc. For this step, we plan to use game testing method as the medium for testing, followed by simple questionnaire to get the feedback of the respondents. This method is often considered as the best and most accurate for getting respondents feedback (Subramoniam, 2015). Figure 14 shows the processes of game testing.

Launch phase: Launch phase consists of releasing the game and supporting activities. This is the phase when the game is ready for presentation to the audience and in this case, ready for presentation to the assessor at the end of the next semester. By this time, the game will need to be completed and run smoothly from start to end. This is a must, to create a great first impression to the audiences. Maintenance phase includes bug fixing and upgrades development. Last but not least, the maintenance phase which is more towards the future planning in a long term. This planning is importance to ensure the project longevity towards the next generation to continues on the project. In this phase, any bug that arises alongside the games release need to be fixed and the content of the game needs to be upgraded and added regularly. More commonly known as expansion packs, this kind of thing is commonly done by lots of developer.

\section{CONCLUSION}

In conclusion as mentioned the above. The objective of this study was to develop a 3D game integrated with
Malaysian identities and values. To do this, the first step was to explore the subject through relevant literature and analyzing proper designs and culture about Malaysia. By following the methodology mentioned above thoroughly, the project will run smoothly till the end. If any hiccups happens, that's a given, it will happen eventually, the most important thing is to keep moving on to the next steps while repairing and fixing what's missing and what's incorrect in the mistake part. A lot was learned from the research that was conducted during the first part of the study and I am glad that I decided to focus much of the time on this part instead of rushing to the actual production. Reading literature by artists and developers in the industry, who explained their procedures and processes of developing video game and designing character based on the main content needs, equipped me with new ideas and views on how to approach the task prior to the actual production. Also, one of the most important aspect in this project is the support and guidance from supervisor and all the relevant lecturer. With their support, this project will end with a success.

\section{ACKNOWLEDGEMENT}

I would like to express my special thanks of gratitude to Allah and my supervisor, Sir Dahlan Abdul Ghani gave me the golden opportunity to do this thesis on the topic Prof.O: exploring Game Engine Attributes to Promote Malaysian Culture which also helped me in doing a lot of research and i came to know about so many new 
things I am really thankful to them. Secondly, I would also like to thank my parents and friends who helped me a lot in finalizing this project within the limited time frame.

\section{REFERENCES}

ABC NEWS, 2011. The benefits of video games. Good Morning America American Television Show, America. https://abcnews.go.com/blogs/technology/ 2011/12/the-benefits-of-video-games/

Abbott, A., 2014. Digital Paper: A Manual for Research and Writing with Library and Internet Materials. University of Chicago Press, Chicago, Illinois, USA., ISBN:9780226167640, Pages: 272.

Alcazaren, P., 2011. Truly colorful Malaysia. Philstar Global, USA.

Amlani, R.D., 2012. Advantages and limitations of different SDLC models. Intl. J. Comput. Appl. Inf. Technol., 1: 6-11.

Anonymous, 2010. SDLC-waterfall model. Tutorials Point (India) Ltd., Hyderabad, India. https://www. tutorialspoint.com/sdlc/sdlc_waterfall_model

Anonymous, 2016. Waterfall model: Advantages \& disadvantages. Study.com., USA. https://study.com/ academy/lesson/waterfall-model-advantagesdisadvantages.html

Anonymous, 2017. Quantitative and qualitative testing. TryMyUi, San Mate, California. https://www. trymyui.com/quantitative-and-qualitative-testing

Bergen, G.V. and D. Gregorius, 2010. Game Physics Pearls. Taylor \& Francis Group, Massachusetts, USA., ISBN:9781568814742, Pages: 344.

Blow, J., 2014. Game development: Harder than you think. Queue, 1: 28-37.

Burton, C., 2015. Adventure creator: Creating a 3D game tutorial. YouTube Video Sharing Company, San Bruno, California, USA.

Byars, M.N., 2015. Printed books versus digital books. BA Thesis, California Polytechnic State University, San Luis Obispo, California, USA.

DSM, 2010. Population distribution and basic demographic characteristic report 2010. Department of Statistics, Malaysia. http://www.statistics.gov.my/ portal/index.php?option $=$ com_content\&view $=$ articl $\mathrm{e} \& \mathrm{id}=1215$.

Granic, I., A. Lobel and R.C.M.E. Engels, 2014. The benefits of playing video games. Am. Psychologist, 69: 66-78.

Gray, C. and M. Julian, 2004. Visualizing Research: A Guide to the Research Process in Art and Design. Ashgate Publishing Limited, Farnham, UK., ISBN:9780754635772, Pages: 214.
HPPST., 2012. Impacts of video games. House of Parliament, Parliamentary of Science \& Technology, London, UK. https://www.parliament.uk/documents/ post/postpn405_impacts_of_videogames.pdf

Iman, M.R., 2011. [Video games are great for kids?]. Utusan Malaysia, Malaysia. (In Malay)

Janlert, L.E. and E. Stolterman, 1997. The character of things. Des. Stud., 18: 297-314.

Jarvi, A., T. Makila and S. Hyrynsalmi, 2013. Game development accelerator-initial design and research approach. CEUR. Workshop Proc., 1: 47-58.

Kimmons, J., 2016. The balance small business. Dotdash, New York, USA. https://www.thebalancesmb.com/

Kraft, G., 2010. Video game testing 101. Ezinarticles, USA. http://yourvideogametesting101.blogspot.com/

Kumar, R., 2011. Research Methodology: A Step by Step Guide for Beginners. 3rd Edn., SAGE, Newcastle Upon Tyne, UK., ISBN:9781446244777, Pages: 440.

Manninen, T., T. Kujanpaa, L. Vallius, T. Korva and P. Koskinen, 2006. Game production process: A preliminary study. MSc Thesis, University of Oulu, Oulu, Finland.

Matney, L., 2017. Apple's revenue growth slows as iPhone sales dip 12\% year-over-year. TechCrunch, Bay Area, USA. https://techcrunch.com/

McGonigal, J., 2015. How video games can teach your brain to fight depression. Slate Magazine, USA. https://slate.com/technology/2015/11/how-videogames-can-teach-your-brain-to-fight-depression.html

Munassar, N.M.A. and A. Govardhan, 2010. A comparison between five models of software engineering. IJCSI, 5: 95-101.

Nantes, A., R. Brown and F. Maire, 2008. A framework for the semi-automatic testing of video games. Proceedings of the 4th Artificial Intelligence Conference on Interactive Digital Entertainment Conference, October 22-24, 2008, The AAAI Press, Menlo Park, California, USA., pp: 197-202.

Pixar, 2012. The typical pixar animation process. Pixar Computer Animation Company, Emeryville, California, USA. http://pixaranimatedfilms.blogspot. com/2017/12/the-typical-pixar-animationprocess.html

Ramadan, R. and B. Hendradjaya, 2014. Development of game testing method for measuring game quality. Proceedings of the International Conference on Data and Software Engineering (ICODSE), November 26-27, 2014, IEEE, Bandung, Indonesia, ISBN:978-1-4799-8175-5, pp: 1-6.

Ruuska, E., 2015. Quality assurance testing on video games: The importance and impact of a misunderstood industry. Master Thesis, Tampere University of Applied Sciences, Tampere, Finland. 
Scacchi, W., 2012. The future of research in computer games and virtual world environments. MSc Thesis, Institute for Software Research, University of California Irvine, California, USA.

Serebrin, J., 2017. Independent video game studios are on the rise. The Globe and Mail Inc., King Street East, Toronto, Ontario. https://beta.theglobeandmail.com/ report-on-business/small-business/sb-growth/ independent-video-game-studios-are-on-the-rise/ article33700472/?ref=http://www.theglobeandmail. com\&

Subramoniam, V., 2015. Game testing: Play pause test. PIT Solutions AG., Thiruvananthapuram, India. https://www.pitsolutions.ch/blog/game-testing-playpause-test/

Sungar, E., 2014. Importance of doing puzzles with your kids. HuffPost, UK.

Taylor, N., C. Kampe and K. Bell, 2015. Me and Lee: Identification and the play of attraction in the walking dead. Game Stud., 15: 1-15.

UCB., 2015. Using the internet for research. University of Colorado Boulder, Boulder, USA. https://www.colorado.edu/history/undergraduates/p aper-guidelines/using-internet-research
UFS., 2009. Module 6: The importance of research. Unite For Sight, New Haven, USA. http://www. uniteforsight.org/research-methodology/module6

UND., 2008. Documenting teaching: Use multiple sources of evidence. University of North Dakota, North Dakota, USA.

Vincent, J., 2017. 99.6 percent of new smartphones run android or iOS. The Verge, New York, USA. https://www.theverge.com/2017/2/16/14634656/an droid-ios-market-share-blackberry-2016

Wang, A.I. and B. Wu, 2015. Use of Game Development in Computer Science and Software Engineering Education. In: Computer Games and Software Engineering, Kendra, M.L.C. and W. Scacchi (Eds.). CRC Press, Boca Raton, Florida, USA., ISBN:9780429159497, pp: 31-58.

Wi, J.H., 2008. Management of an online game development project. Ann. Bus. Administrative Sci., 7: 33-44.

Wisdom, J. and J.W. Creswell, 2013. Mixed methods: Integrating quantitative and qualitative data collection and analysis while studying patient-centered medical home models. Agency for Healthcare Research and Quality, Rockville, Maryland, USA. https://pcmh.ahrq.gov/page/mixedmethods-integrating-quantitative-and-qualitativedata-collection-and-analysis-while 